\title{
Active power measurement verification for electric power systems with battery energy storage
}

\author{
Anna Glazunova ${ }^{1, *}$, Elena Aksaeva ${ }^{1}$ \\ ${ }^{1}$ Melentiev Energy Systems Institute of Siberian Branch of the Russian Academy of Sciences
}

\begin{abstract}
The paper presents a method developed for detecting rough errors in the measurements related to batteries in the part of electric power system that is characterised by low data redundancy. Since batteries either produce or consume power, not all methods of bad data detection can be used to detect erroneous measurements of the active power of the battery in the case of low measurement redundancy. Because of different values of the active power that a battery may produce or consume at several snapshots in row, dynamic algorithms cannot be used. In this study, a new method of bad data detection is developed. The method is based on the battery control strategy analysis.
\end{abstract}

\section{Introduction}

The main objectives in managing an electric power system (EPS) with renewable energy sources are to mitigate fluctuations in the system and to accept as much solar and wind power as possible. These objectives can be met by using energy storage devices. There are lots of energy storage technologies:

Pumped hydro energy storage (PHES);

Compressed air energy storage (CAES);

Hydrogen;

Batteries;

Flywheels;

Superconducting magnetic energy storage;

Supercapacitors.

There are several technologies of Pumped hydro energy storage (PHES) in Russia. Currently, much attention is paid to large-scale intermediate energy storage in power grids of various levels. Energy is stored using energy storage technologies, which allow smoothing energy consumption over time, shave peak loads and, thereby, reduce the required operating power of the main equipment of the system.

Currently there is no energy storage technology that can provide a long lifetime, low cost, and high efficiency at the same time [1], [2].

One of the most common energy storage devices are batteries.

The paper is organized as follows. The second section presents a description of the problem. The focus of the third section is a state estimation method. The fourth section explains a technique of the developed method. The fifth section describes modeling of wind speed and wind power generation. The sixth section several case studies are presented. The last section presents the conclusions.

\section{Description of the problem}

In electric power system control, validity and completeness of data are provided by the state estimation procedure [3], [4], which calculates steady-state variables on the basis of a measurement snapshot (measurements obtained at one and the same time point). High quality of estimates is ensured when rough errors in measurements are detected and eliminated in advance.

Rough errors can be detected and eliminated in the stage of bad data detection [5], [6], [7] provided there is sufficient redundancy of the measurements. Integration of renewable energy sources and energy storage systems into the electric power system potentially causes low measurement redundancy. Under such conditions, conventional bad data detection methods do not work, especially when wind and solar generation changes rapidly.

Batteries supply or consume power. That is why not all methods of bad data detection can be used to detect erroneous measurements of the active power of the battery under low measurement redundancy. Because of different values of the active power that a battery may produce or consume at close intervals (two snapshots in a row), dynamic algorithms cannot be used.

In this study, a method of bad data detection is based on the battery control strategy analysis according to which the battery is discharged when there is a shortage of power generated at the wind farm, and it is charged when there is too much power generated at the wind- farm.

\section{State estimation}

State estimation of an electric power system implies calculation of state variables, and is based on the

Corresponding author: glazunova@isem.irk.ru 
measurements obtained from SCADA system (Supervisory Control and Data Acquisition) and WAMS (Wide-Area Measurement Systems).

The vector of measurements looks as follows:

$$
\bar{y}=\left(U_{i}, P_{i}, Q_{i}, P_{i j}, Q_{i j}, \delta_{i}\right),
$$

where $\quad P_{i}=P_{l(i)}+P_{g(i)}, \quad Q_{i}=Q_{l(i)}+Q_{g(i)}$,

$U_{i}$ - magnitudes of nodal voltages; $P_{g(i)}, Q_{g(i)}$-active and reactive power generation at nodes; $P_{l(i)}, Q_{l(i)}-$ active and reactive power load at nodes, $P_{i j}, Q_{i j}-$ power flows in transformers and lines, $\delta_{i}$ - voltage phases at the nodes with PMUs (Phasor Measurement Units).

The objective function in the state estimation is as follows:

$$
J(x)=(\bar{y}-y(x))^{T} R_{y}^{-1}(\bar{y}-y(x)),
$$

where $R_{y}$-a covariance matrix of measurement errors, $x=(U, \delta)$ - state vector (a part of the state variables that is used to calculate all the rest state variables).

In order to calculate the state vector, the derivative $\frac{\partial J(x)}{\partial x}$ is equated to zero, and the system of non-linear equations is solved:

$$
H^{T} R^{-1}(\bar{y}-y(x))=0,
$$

where $H=\frac{\partial y}{\partial x}-$ Jacobi matrix.

The system (3) is linearized at each iteration $i$. The system of linear equations is solved, where correction vector is calculated by the equation:

$$
\begin{gathered}
\Delta x^{(i)}=P^{(i)}\left(H^{T(i)} R_{y}^{-1}\left(\bar{y}-y\left(x^{(i)}\right)\right),\right. \\
P^{(i)}=\left(H^{T(i)} R^{-1} H^{(i)}\right)^{-1},
\end{gathered}
$$

where $P^{(i)}$ - a covariance matrix of state vector estimation errors.

Components of the state vector are calculated by the equation:

$$
x^{(i+1)}=x^{(i)}+\Delta x^{(i)},
$$

If $\Delta x<d_{x}$, the iteration process converges, where $d_{x}$ - accuracy of the iterative process. Then, a load flow solution is obtained using the state vector and values of generation and load at each node are calculated.

\section{The proposed method of bad data detection}

In this study, a method of bad data detection is developed. A rough error in a measurement of active power produced or consumed by a battery is detected. The basic idea of the method is as follows: the measurement of the active power produced or consumed by the battery $\bar{P}_{B}$ must coincide with the value of the active power calculated in accordance with the battery control strategy $P_{B}$ :

$$
\bar{P}_{B}=P_{B} \text {. }
$$

The calculated value of active power is used to determine the state of charge, which cannot exceed $100 \%$. The method consists of two steps. Fig. 1 shows a bad data detection algorithm.

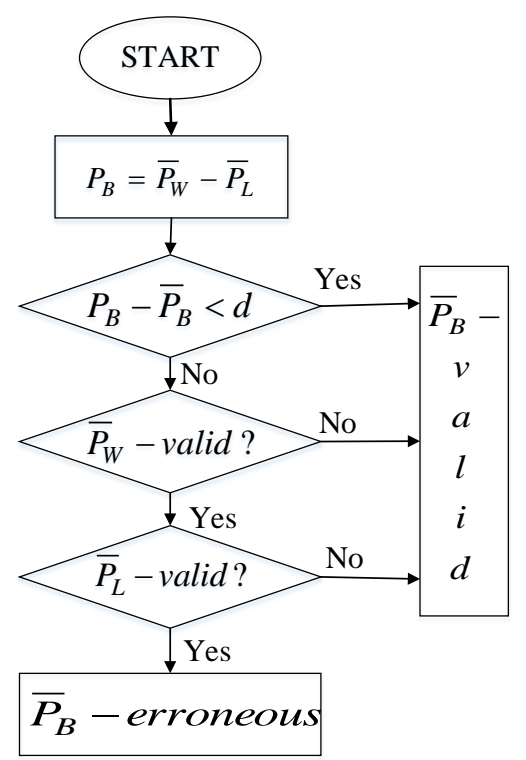

Fig.1. Bad data detection algorithm

The first step. The active power produced or consumed by the battery is calculated in accordance with the strategy described in [8]. In this study the active power is calculated by the formula:

$$
P_{B}=\bar{P}_{W}-\bar{P}_{L},
$$

subject to

$$
S O C_{\min }<S O C(t)<1,
$$

where $\bar{P}_{W}$ - the active power generation measurement at wind farm, $\bar{P}_{L}$ - the measurement of load, $\operatorname{SOC}(\mathrm{t})$ - state of charge at moment $t$ is calculated in [1] in terms of its initial value. In this study, SOC is calculated by the formula

$\operatorname{SOC}(t+\Delta t)=\operatorname{SOC}(t)-\frac{1}{W_{C A P}}\left(P_{B}+P_{L s}\right) \Delta t \cdot$ status

where:

$W_{C A P}$ - battery capacity (MWh) (given),

$P_{B}$ - power consumed or supplied by the battery (given),

$P_{L s}-$ self needs of the battery energy storage system;

$\Delta t$-duration,

status -0 or 1 (connected or disconnected) (given),

$S O C_{\text {min }}-$ minimum value of SOC.

To detect a rough error in the measurement of active power, the inequality is analyzed. 


$$
\begin{gathered}
\left|P_{B}-\bar{P}_{B}\right|<d, \\
d=3 \sqrt{\sigma_{B}^{2}+\sigma_{W}^{2}+\sigma_{L}^{2}},
\end{gathered}
$$

where $\sigma_{B}^{2}$ - the variance of the measurement of active power that is produced or consumed by the battery, $\sigma_{W}^{2}-$ the variance of the measurement of active power at the wind farm, $\sigma_{L}^{2}$ - the variance of the measurement of active power at the load.

Failure (11) means that there is an error either in a measurement of active power produced or consumed by a battery or in other measurements related to (11).

The second step is needed to detect rough errors in measurements $\bar{P}_{W}$ and $\bar{P}_{L}$, when inequality (11) is not satisfied.

\section{Modeling of wind speed and wind power generation}

In this study, Weibull distribution [9] is used to estimate the probability of wind speed. Figure 2 shows that the wind turbine starts to work at wind speed $V_{\text {cin }}=$ $5 \mathrm{~m} / \mathrm{s}$ and reaches rated power at wind speed $V_{r}=$ $12 \mathrm{~m} / \mathrm{s}$. Then, despite the increase in wind, the wind turbine power does not increase until $V_{c c}=26 \mathrm{~m} / \mathrm{s}$ is reached. Figure 3 shows the probability of wind speed which is estimated by the Weibull distribution at $\mathrm{a}=12.2$, $\mathrm{b}=6$, where $\mathrm{a}, \mathrm{b}$ are constants used in Weibull distribution.

Figure 4 demonstrates a curve of active power of wind generator, that is calculated on the basis of the typical curve of a wind turbine (figure 2) and on the basis of the curve of wind speed (figure 3 ) by the formula:

$$
P_{W}=1 / 2 \rho A v^{3} c_{p},
$$

where $\rho$ - the density of air $(1.605 \mathrm{~kg} / \mathrm{m} / \mathrm{m}), v$ - the wind speed, $A$ - the rotor area $\left(1124 \mathrm{~m}^{2}\right), c_{p}$ - the power coefficient.

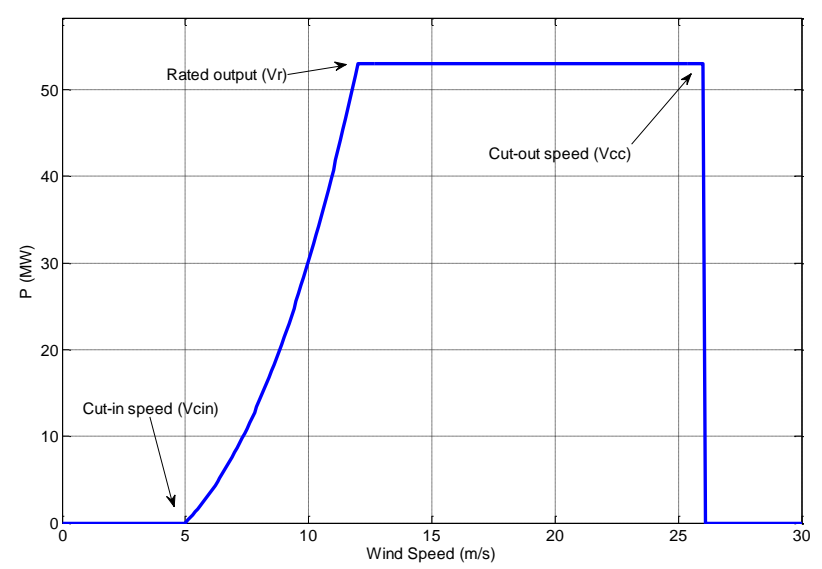

Fig. 2. A typical curve of wind turbine

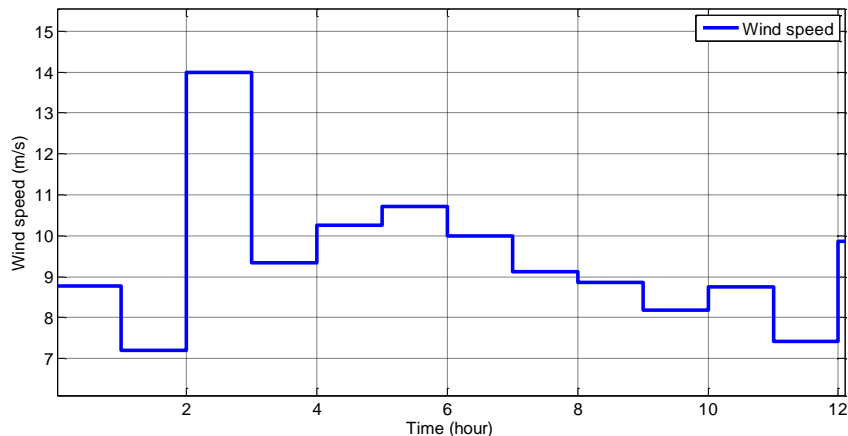

Fig.3. Wind speed

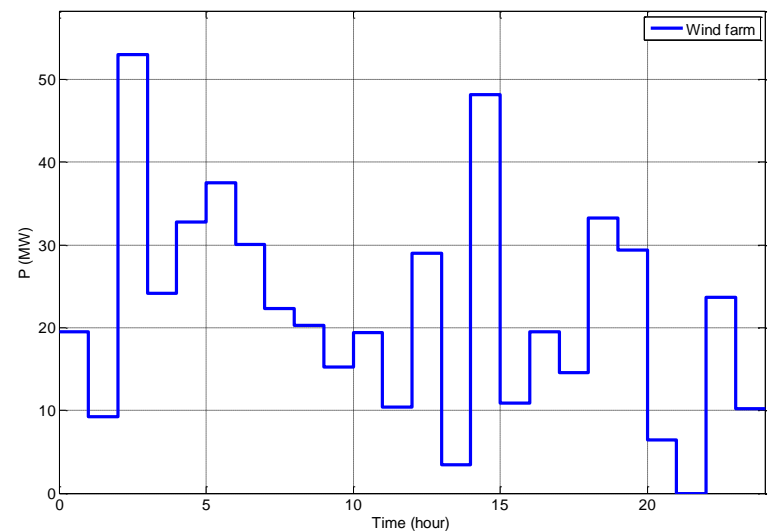

Fig. 4. Active power of wind turbine

\section{Case study}

\subsection{Archive of measurements and scenario}

An archive of conditions is created on the basis of the mathematical model of a 5-bus test system (Figures 5, 6) under various operating conditions. A load flow solution for each point of the load curves is used to create measurement snapshots. The measured state variables of a particular solution are changed by adding a small random value according to the model of measurement

$$
\bar{y}=y_{\text {true }}+x_{\text {rand }} \sigma_{y},
$$

where $\sigma_{y}$ - measurement standard deviation, $\sigma_{y}^{2}$ is a measurement error variance determined on the basis of the characteristics of the metrological path, $x_{\text {rand }}$ - random errors generated by random number generator, $x_{\text {rand }} \in N(0,1), y_{\text {true }}$ - reference (a load flow solution).

The calculations are aimed at detecting rough errors in the measurement of active power produced or consumed by a battery $\left(P_{5}\right)$ and in the measurements related to nodes 1,3 .

$$
P_{w(\max )}=14.8 M W, W_{C A P}=7 M W h, P_{L}=15 M W \text {. }
$$


$P_{w(\max )}-$ maximum active power generated by the wind farm, $P_{L}-$ load.

This study presents two cases. The first case: the measurements $\left(\bar{P}_{1}, \bar{P}_{5}\right)$ are included in a group of measurements with low redundancy (Figure 5). This means on the one hand that $\bar{P}_{1}, \bar{P}_{5}$ are not critical because other measurements are available, but on the other hand $\bar{P}_{1}, \bar{P}_{5}$ remain untested because of the impossibility of making up an equation of active power balance at nodes 1 and 5. Measurement $\bar{P}_{3}$ is redundant. This means that it is possible to make up the equation of balance at node 3 .

The second case: the measurements $\vec{P}_{3}, \bar{P}_{5}$ are redundant (Figure 6). This means that it is possible to make up the equation of balance at nodes 3 and 5 . Measurement $\bar{P}_{5}$ has low redundancy.

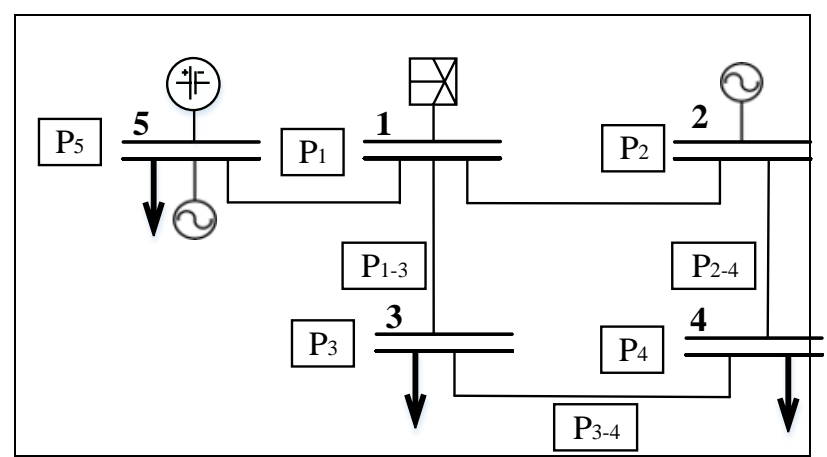

Fig.5. Test system. Low data redundancy $\left(P_{1}, P_{5}\right)$

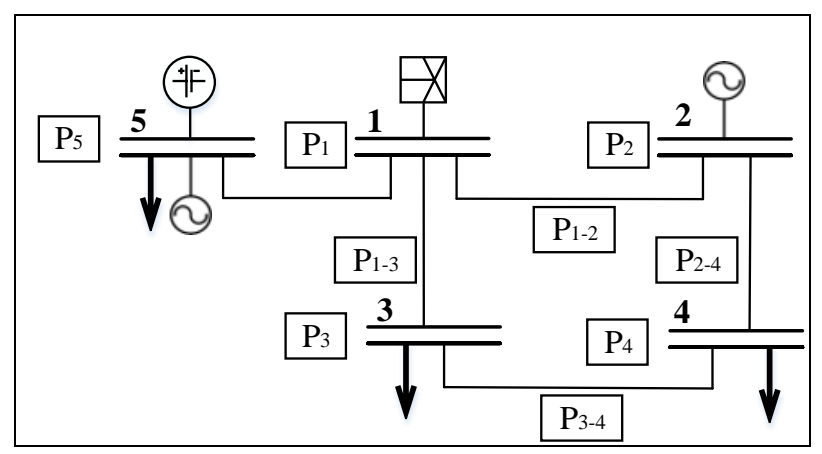

Fig.6. Test system. Low data redundancy $\left(P_{5}\right), P_{1}-$ redundant

\subsection{Strategy of the battery controller}

Figures 7 and 9 show active power curves (battery, wind power, load, a sum of battery power and wind power) obtained when the strategy of the controller is as follows: with insufficient power generated at the wind farm, the battery is discharged, when there is surplus power generated at the wind farm, the battery is charged. Figures
7 and 9 show the references and measurements of these values, respectively. Figures 8 and 10 illustrate the references and a real value of the SOC (state of charge).

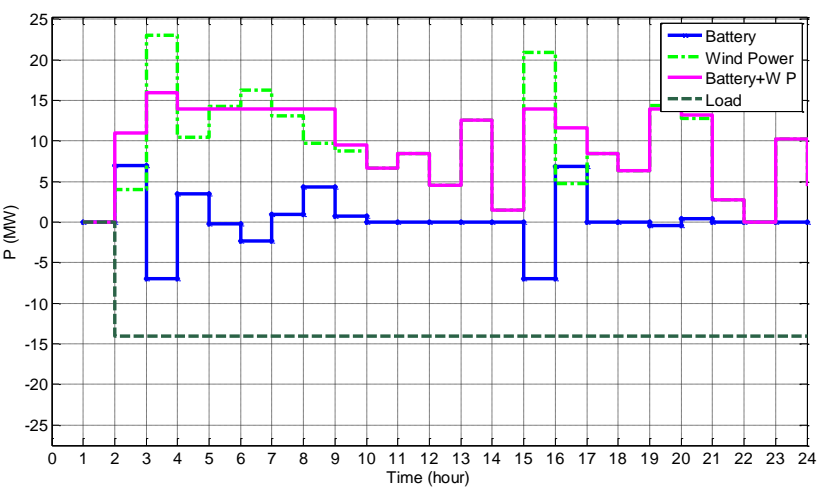

Fig.7. Reference values of active power

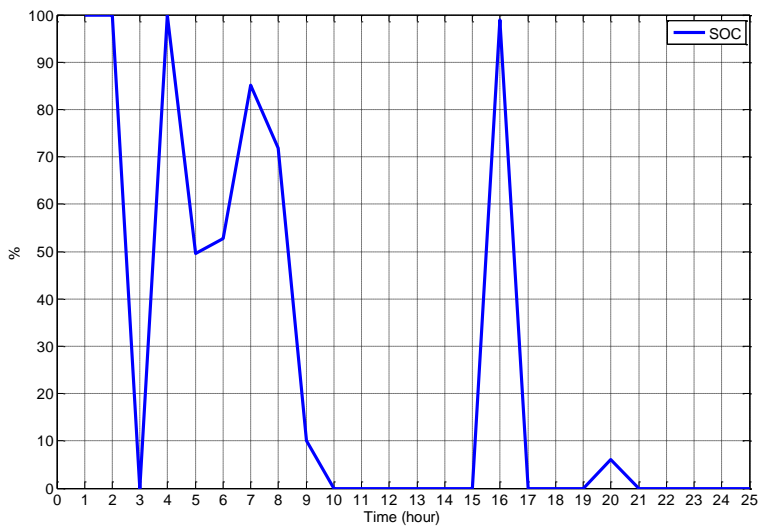

Fig.8. Reference values of SOC

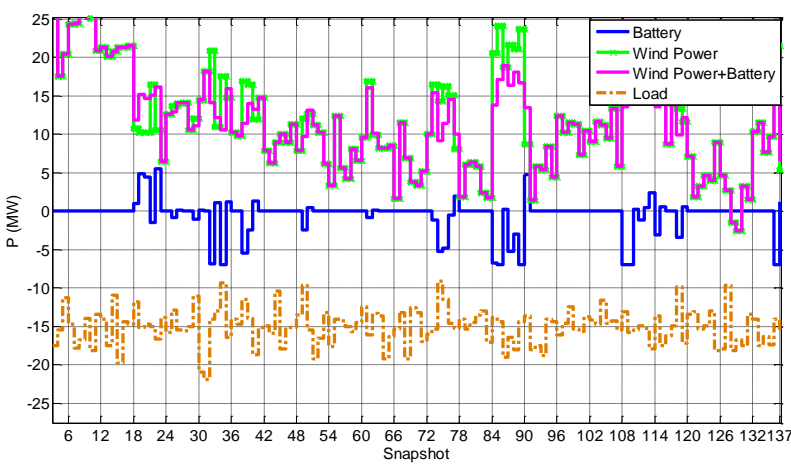

Fig.9. Curves of active power measurement (at the battery references)

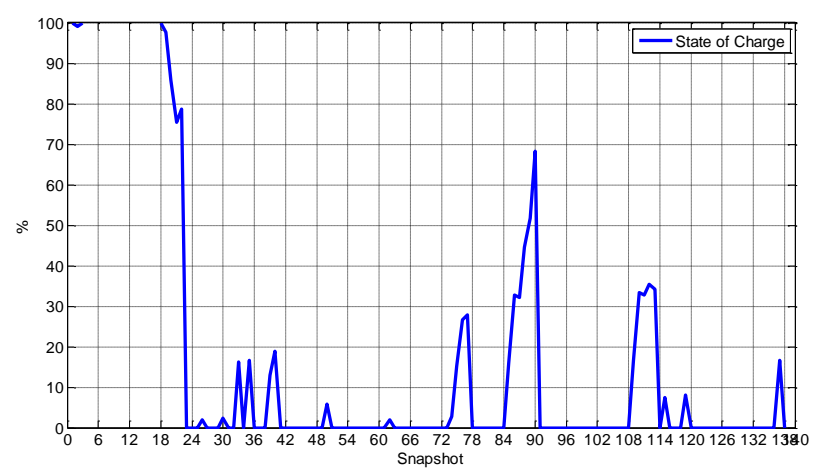

Fig.10. State of charge (140 snapshots) 
Figure 8 shows that the battery is fully charged from 3.00 to 4.00 and from 15 to 16 , i.e. the wind farm produces enough power to cover the load. From 10.00 to 15.00 , from 17.00 to 19.00 and from 21.00 to 24.00 , the battery is completely discharged.

\subsection{Simulation of a rough error and description of experiments}

A rough error in the measurement is simulated by the formula

$$
\bar{y}=y_{\text {true }}+x_{\text {ran }} \sigma_{i} n,
$$

where $\mathrm{n}$ is used to simulate a rough error that varies in magnitude.

There are three experiments in this study which assume that:

1. The active power value determined in accordance with the battery strategy is accurate. The threshold is calculated by the formula:

$$
d=3 \sqrt{\sigma_{B}^{2}}
$$

2. The active power value is determined taking into account random errors in measurements. Their variances are approximately equal to each other. The threshold is calculated by the formula (12).

3. The active power value is determined taking into account random errors in measurements. Their variances differ from each other. The threshold is calculated by formula (12).

As an example, a rough error is modeled in measurement $\bar{P}_{5}$ by the formula:

$$
\bar{y}=y_{\text {true }}+x_{\text {ran }} \sigma_{i} 4 \text {. }
$$

The rough errors are added to the measurements of active power in the battery in snapshots $23,35,50$. In figure 11 snapshots $(23,35,50)$ that have an erroneous measurement of active power at the battery are marked.

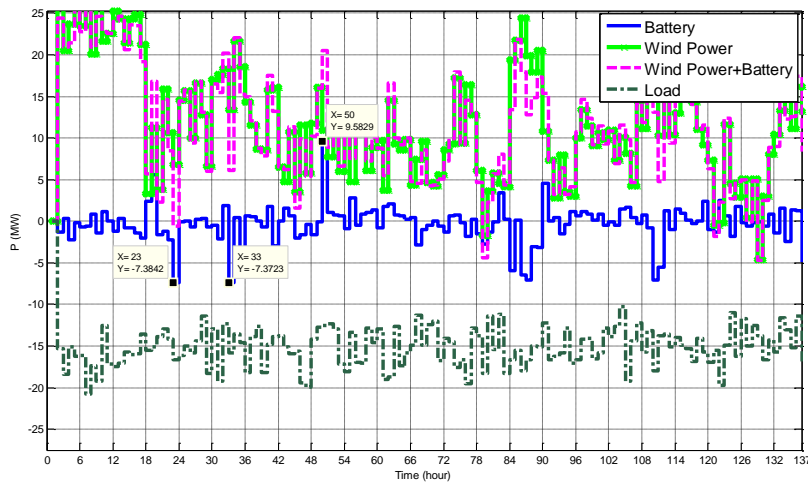

Fig.11. Curves of active power (measurement at the battery has an error in snapshots $23,35,50$ )

Case 1. Step 1. Active power is calculated by formula (8) (snapshot 23)

$$
P_{B}=8.6 M W
$$

Condition (11) is analyzed. Inequality (11) that looks as follows

$$
|8.6+7.3|<3.67 \quad \text { is false. }
$$

The threshold $d$ calculated by formula (12) is equal 3.67 MW.

The answer "false" means that there is an error either in the active power measurement at wind farm or in the active power measurement of the battery or in the measurement of load.

Step 2. To verify measurement $\bar{P}_{3}$, the equation of active power balance at node 3 is made up

$$
P_{3}+P_{1-3}+P_{3-4}=0 \text {. }
$$

To verify measurement $\bar{P}_{1}$, the problem of the rough error detection in an active power measurement at wind farm is solved using the algorithm developed in [10] Depending on the results of verification of measurements $\bar{P}_{3}, \bar{P}_{5}$, the active power measurement of the battery is considered to be either erroneous or valid.

Case 2. Step 1. Active power that the battery may produce or consume is calculated by (8). Then inequality (11) is analyzed.

Step2. To verify the reliability of measurement $\bar{P}_{1}$, the active power balance equation is compiled at node 1 .

$$
P_{5}+P_{1}+P_{1-2}+P_{1-3}=0 \text {. }
$$

The reliability of measurement $\bar{P}_{3}$ is tested using equation (20).

\subsection{Results}

The results obtained in experiments $1,2,3$ are shown in table 1 and in figures 12,13,14.

Table 1. The number of errors detected (\%)

\begin{tabular}{|c|c|c|c|c|c|}
\hline \multirow{2}{*}{$\begin{array}{c}\mathrm{N} \\
\text { experim }\end{array}$} & \multicolumn{5}{|c|}{$\mathrm{n}$ (in formula 15) } \\
\cline { 2 - 6 } & 4 & 5 & 8 & 10 & 12 \\
\hline 1 & 100 & & & & \\
\hline 2 & 67 & 100 & & & \\
\hline 3 & 0 & 0 & 33 & 67 & 100 \\
\hline
\end{tabular}

Experiment 1 .

Figure 12 shows the number of erroneous snapshots. Figure 12 shows that all rough errors modeled with $n=4$ were detected.

Experiment 2.

An analysis of results indicates that if the variances of the measurements are approximately equal to each other ( $\sigma_{B}^{2}=4.5 M W^{2}, \sigma_{W}^{2}=5.1 M W^{2}, \sigma_{L}^{2}=5.0 M W^{2}$ ), rough errors can be detected using the developed method with $\mathrm{n}=5$ and more.

Experiment 3.

Figures 13 and 14 show that with $n=8$, and $n=10$ one third and two thirds of the simulated errors can be detected, respectively. 
An analysis of the results shows that if the variances of the measurements strongly differ from each other

$\left(\sigma_{B}^{2}=1.5 M W^{2}, \sigma_{W}^{2}=10.1 M W^{2}, \sigma_{L}^{2}=5.0 M W^{2}\right)$

the developed method allows detecting rough error with $\mathrm{n}=12$ and more.

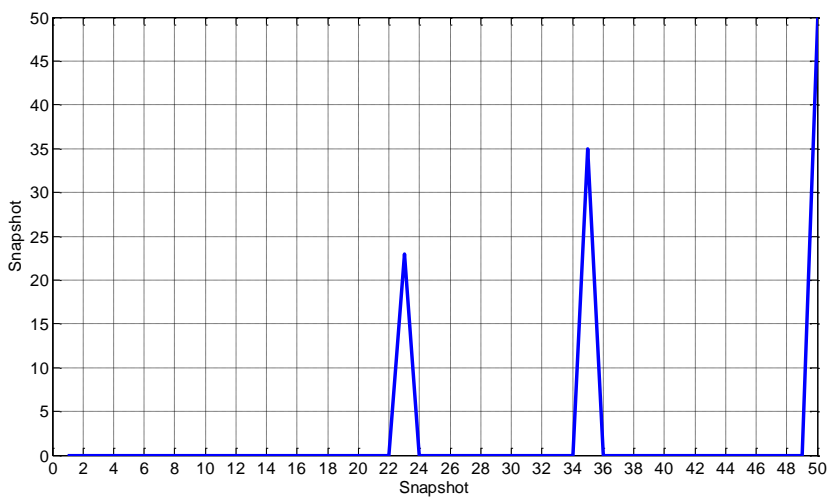

Fig.12. The number of erroneous snapshots (experiment 1, $\mathrm{n}=4$ )

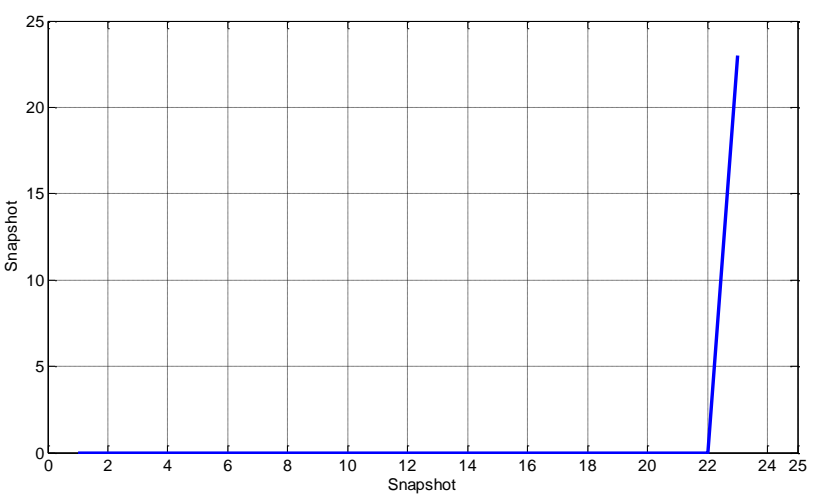

Fig.13 The number of erroneous snapshots (experiment 3, $\mathrm{n}=8$ )

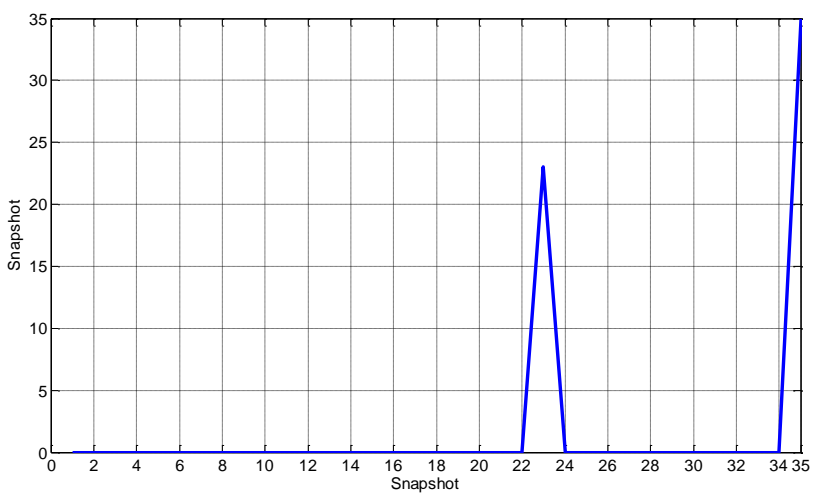

Fig.14 The number of erroneous snapshots (experiment $3, \mathrm{n}=10$ )

\section{Conclusion}

A new method of active power measurement verification for electric power systems with battery energy storage is presented. The method is based on the battery control strategy analysis.

Analysis of the results shows that depending on the method of calculating the threshold (depending on the ratio between the variances of measurements), the magnitude of the rough error that can be detected using the developed method is different.

The results of experiments 1, 2, 3 show that the method developed in this study can be used to detect rough errors with $n=4$ (not high error), $n=5$ (middle error), and $n=12$ (high error), respectively.

This study is supported by grant № 19-49-04108. "Development of Innovative Technologies and Tools for Flexibility Assessment and Enhancement of Future Power Systems".

\section{References}

1. Kanzumba Kusakana, Optimal scheduled power flow for distributed photovoltaic/wind/diesel generators with battery storage system (The institution of engineering and technology, V.9, No. 8, pp. 916-924, 2015)

2. Peter D. Land, Juuso Lindgren, Jani Mikkola, Juri Salpakari, Review of energy system flexibility measures to enable high levels of variable renewable electricity (Renewable and sustainable energy reviews, V. 45, pp. 785-807, 2015)

3. A.M. Glazunova, I.N.Kolosok, E.S.Korkina, Monitoring of EPS operation by the state estimation methods (Innovative Smart Grid Technologies (ISGT Europe-2011), Great Britain, Manchester, 2011)

4. Ali Abur, Antonio Gomez Exposito, Power system state estimation: Theory and implementation (New York, : NY: Marcel Dekker, 2004, p. 327)

5. Fantin C.A., Massignan J.A.D., Castillo M.R., London J.B.A., Observability, redundancy and gross error processing in state estimation using SCADA and synchronized phasor measurement (Proceedings of the International Conference PowerTech, Eindhoven, Netherlands, 2015)

6. Pignati M., Zanni L., Sarri S., Cherkaoui R., Boudec J.-Y. Le, Paolone M., A pre-estimation filtering process of bad data for linear power systems state estimation using PMUs (Proceedings of the 18th Power Systems Computation Conference, Wroclaw, Poland, 2014)

7. A.M. Glazunova, I.N. Kolosok, E.C. Korkina, Study of Test Equations Method's Application for Bad Data Detection in PMU Measurements (Proceedings of PMAPS, Istanbul, Turkey, 2012)

8. Sercan Teleke, Mesut E. Baran, Subhashish Bhattacharya, Alex Q. Huang, Rule-based control of battery energy storage for dispatching intermittent renewable sources (IEEE Transactions on sustainable energy, V. 1, No. 3, pp. 117-124, 2010)

9. Internet. http://xn--drmstrre-64ad.dk/wpcontent/wind/miller/windpower\%20web/en/tour/wre s/weibull.htm

10. Glazunova A., Aksaeva E, «An Increase in Information Security of Electric Power System with Wind Power Penetration under Low Redundancy of Measurements (Proceedings of PowerTech, Milan, Italy, 2019) 